\title{
Styrene Cross-metathesis Using Low Catalyst Concentrations
}

\author{
Paul Sabila \\ Department of Science, Technology, and Mathematics, Chemistry and Physics Program, Gallaudet University, United States
}

Copyright (C) 2015 by authors, all rights reserved. Authors agree that this article remains permanently open access under the terms of the Creative Commons Attribution License 4.0 International License

\begin{abstract}
A new study reports on the use of low concentrations of metathesis catalysts $(\sim 0.009 \mathrm{~mol} . \%)$ in the cross-metathesis of styrene to afford relatively good yields $(87 \%)$ of trans-stilbene (the cross-metathesis homodimer product). The reaction was carried out under aerobic conditions after 3-5 h reflux. Also, a theoretical prediction of the cross-metathesis product at ultra-low concentrations is presented.
\end{abstract}

Keywords Cross-metathesis, Styrene, Catalysis

\section{Introduction}

Over the last two decades, great strides have been made in the exploitation of the olefin metathesis reactions. Most of the reported developments have capitalized on the metathesis reactions' powerful carbon-carbon bond-formation and carbon-carbon bond-breaking transformations to effect coupling, cyclization, ring opening and macrocyclization reactions. ${ }^{1,2}$ Metathesis reactions have also been employed to effect further substrate functionalization enabling more complex structures to be prepared in a few steps. ${ }^{3}$ The metathesis transformations widely used in organic synthesis include ring closing metathesis (RCM), cross metathesis (CM), ring opening metathesis (ROM) and ring opening metathesis polymerization (ROMP). The most common catalysts used for these transformations include Schrock, ${ }^{4}$ Grubbs $1^{\text {st }}$ generation (1), ${ }^{5}$ Grubbs $2^{\text {nd }}$ generation (2), ${ }^{6}$ Hoveyda-Grubbs $1^{\text {st }}$ generation (3), and Hoveyda-Grubbs $2^{\text {nd }}$ generation (4) (see Fig. 1). In particular, catalysts 1-4 are more widely used in alkene metathesis due to their versatility and stability under a wide range of experimental conditions. In addition, metathesis transformations using these catalysts are generally unaffected by the presence of various functional groups including carbonyls, aromatic rings, amines and alcohols among others thereby eliminating the need for protecting groups ${ }^{7}$ though some amines may require initial conversion to ammonium salts ${ }^{8}$ prior to the metathesis step. Various solvents have been used in metathesis reactions, the most common ones being dichloromethane (when lower reaction temperatures are desired) and toluene (higher reaction temperatures). Metathesis reactions have also been shown to proceed in other solvents, including water ${ }^{9}$ and ionic liquids. ${ }^{10,11}$ The work-up process of metathesis reactions are generally straight forward, the most common method of purification involve filtration through a pad of silica gel to trap the catalyst. This is usually employed for quantitative transformations or for cases where the side-products and unreacted starting materials are sufficently volatile. In cases where significant amounts of non-volatile side-products are present, the reaction mixture is usually concentrated under vacuum and then purified by silica gel column chromatography.

Contrary to expectations, pharmaceutical industries have been generally reluctant to embrace metathesis transformations, in part, due to difficulties in removing or recycling the catalysts after the reaction. ${ }^{12,13}$ Processes that employ reduced catalyst loading could be more attractive as it could significantly reduce the amount and cost of the metathesis catalysts required for industrial-scale transformations. Also, the use of low catalyst loading leads to reduced concentrations of heavy metals in final products leading to a greener and more sustainable synthesis protocols. $^{14}$<smiles>Cl[R](Cl)([Pb])C=[As]</smiles>

1

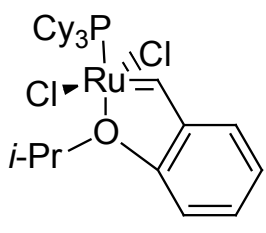

3

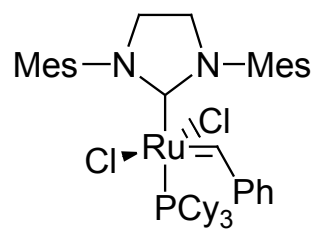

2
Figure 1. Commonly used metathesis catalysts. 
To date, various cases of low catalyst loading have been reported in several metal-catalyzed transformations. High turnover effective numbers (TON) for ruthenium-based metathesis reactions have been reported in solvent-free conditions. ${ }^{15}$ These reactions were carried out under inert conditions. ${ }^{16,17}$ Under these conditions, catalyst 2 was reported to give a TON of 95,000 with a conversation rate of $65 \%$ to afford a CM trans-product with over $91 \%$ selectivity. ${ }^{15}$

Our group was interested in studying the effect of low catalyst loading of I, 2, 3 and $\mathbf{4}$ in cross metathesis of styrene. At the same time, we were interested in comparing the reactivity of the four catalysts during the $\mathrm{CM}$ of styrene. We chose styrene for the current study (we later plan to study other alkene systems) since styrene $\mathrm{CM}$ to give trans-stilbene $\mathbf{5}$, is a well-known reaction and any unreacted styrene is easily separated from the product by column chromatography. According to the classification method developed by Grubbs, highly reactive olefins that readily undergoes homodimerization are classified as Type I while those that are least reactive ordo not undergo homodimerization or $\mathrm{CM}$ are classified as Type IV. ${ }^{18}$ Styrene is classified as Type II with respect to catalyst $\mathbf{1}$ and Type I with respect to catalyst $2 .{ }^{18}$ A literature search did not reveal similar classification with respect to Hoveyda-Grubbs catalysts 3 and $\mathbf{4}$. In addition, there were no literature reports, At the time of our study, there were no reports of CM of styrene using catalysts 3 or 4 .

\section{Metathesis Reaction}

\subsection{Styrene Cross-metathesis Optimization}

As expected, styrene was found to readily undergo homodimerization to afford trans-stilbene in the presence of catalyst 2 (Fig. 2) (no attempt made to use inert atmosphere). Most metathesis reactions reported in the literature employs 2.5-10 mol. \% of metathesis catalysts. Grubbs and co-workers reported a yield of $94 \%$ of trans-stilbene 5 when the 2.5 mol. $\%$ catalyst 2 was used. ${ }^{18}$

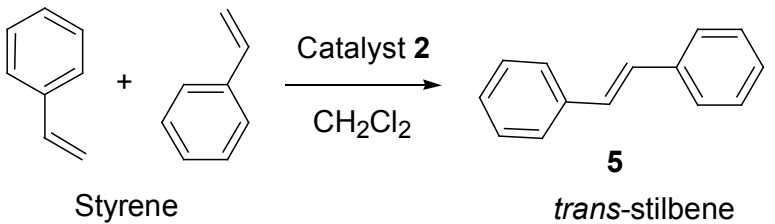

Figure 2. Cross-metathesis of styrene.

In our study, the progress of the $\mathrm{CM}$ of styrene was monitored by TLC and the spots visualized under UV-light. It is important that the UV visualization is done immediately since delay allows the volatile styrene spot (blurred spot) to quickly evaporate from TLC plates leaving the stilbene product as the only UV-active spot. The formation of the trans-stilbene product was also monitored by GC-MS. Finally, the isolated product was characterized by ${ }^{1} \mathrm{H}$ NMR and ${ }^{13} \mathrm{C}$ NMR.

Optimum conditions: A study was done to determine the optimum conditions for the styrene CM reaction. In order to do this, the reaction was run in dichloromethane using catalyst $\mathbf{2}$ at room temperature (RT) and at reflux while monitoring by TLC and GC-MS. A preliminary sampling of the reaction conditions revealed that quantitative amount of cross-metathesis product was obtained when the reaction was run under reflux for at least $3 \mathrm{~h}$ using $1 \mathrm{~mol} \%$ of catalyst 2 as shown on Table 1. The ${ }^{1} \mathrm{H}$ NMR and ${ }^{13} \mathrm{C}$ NMR Spectra of 5 matched with that reported in the literature. ${ }^{19,20}$

Table 1. Optimization of CM reaction using catalyst 2.

\begin{tabular}{|c|c|c|c|}
\hline (mol. \%) & Time $(\mathrm{h})$ & Conditions & \% Yield \\
\hline 1 & 24 & RT & Negligible \\
\hline 0.06 & 1 & Reflux & $>50$ \\
\hline 1 & 3 & Reflux & Quantitative \\
\hline
\end{tabular}

\subsection{Effects of Catalyst Loading on Cross Metathesis}

Next, we proceeded with the study of the effect of the four metathesis catalysts 1, 2, 3 and $\mathbf{4}$ on the yield of styrene CM product 5 . The catalyst concentration was kept between 0.02 and $0.2 \mathrm{~mol} . \%$. The results obtained when the reaction time was kept between 3-5 hours are presented (Table 2). Catalyst 3 was the least active, only yielding negligible amounts of the $\mathrm{CM}$ product when $0.2 \mathrm{~mol} \%$. This was followed by catalyst 1 which provided only $24 \%$ of the CM product when $0.02 \mathrm{~mol} \%$ was used. On the other hand, catalyst 4 was found to the second most active yielding $67 \%$ of the $\mathrm{CM}$ product when $0.023 \mathrm{~mol} . \%$ was used. Catalyst 2 was the most promising, giving $95 \%$ yield of the $\mathrm{CM}$ product when 0.4 mol.\% was used.

Table 2. Study of Catalyst Effect on Styrene CM

\begin{tabular}{|c|c|c|c|}
\hline Catalyst & Catalyst (mol. \%) & Time (h) & $\%$ Yield \\
\hline $\mathbf{1}$ & 0.02 & $5 \mathrm{~h}$ & $\sim 24^{\mathrm{a}}$ \\
\hline $\mathbf{2}$ & 0.04 & $3 \mathrm{~h}$ & 95 \\
\hline $\mathbf{3}$ & 0.2 & $3 \mathrm{~h}$ & Negligible \\
\hline $\mathbf{4}$ & 0.023 & $4 \mathrm{~h}$ & 67 \\
\hline
\end{tabular}

a $24 \%$ of conversion

Having determined the superiority of catalyst $\mathbf{2}$, we set out to determine the yield of $\mathrm{CM}$ product when much lower catalysts concentrations were used. The results (Table 3) indicates that catalysts $\mathbf{2}$ is still highly potent even at 0.009 mol.\% giving a reasonable yield $(87 \%)$ of the $\mathrm{CM}$ product after $4 \mathrm{~h}$ or reflux. This catalyst loading is about 270 to 550 times less concentrated than those reported in the literature (2.5-5 mol. \%) for styrene CM.

Table 3. Study of Catalyst 2 Loading Effect on CM

\begin{tabular}{|c|c|c|c|}
\hline Catalyst & Catalyst (mol. \%) & Time (h) & \% Yield \\
\hline $\mathbf{2}$ & 1 & $3 \mathrm{~h}$ & $100^{\mathrm{b}}$ \\
\hline $\mathbf{2}$ & 0.04 & $3 \mathrm{~h}$ & 95 \\
\hline $\mathbf{2}$ & 0.009 & $4.5 \mathrm{~h}$ & 87 \\
\hline
\end{tabular}

${ }^{\mathrm{b}}$ Quantitative amounts of product was obtained. 
Plotting the results obtained from catalyst 2 (Table 3 ) gives an exponential relationship between the \% yield of the $\mathrm{CM}$ product and the mol.\% of the catalyst used (Figure 3). The equation that roughly fits this curve is $\mathbf{y}=\mathbf{1 0 0 . 8 9} \mathbf{x}^{\mathbf{0 . 0 2 7 4}}$ as shown. If this experiment is to be carried out on an industral scale, several parameters will have to be taken into consideration including the costs of running a reaction for longer periods. Under our experiemntal conditions, reasonable yields are obtained within 5 hours of reflux. We have been able to reproduce these results for up to $20 \mathrm{~g}$ scale of styrene.

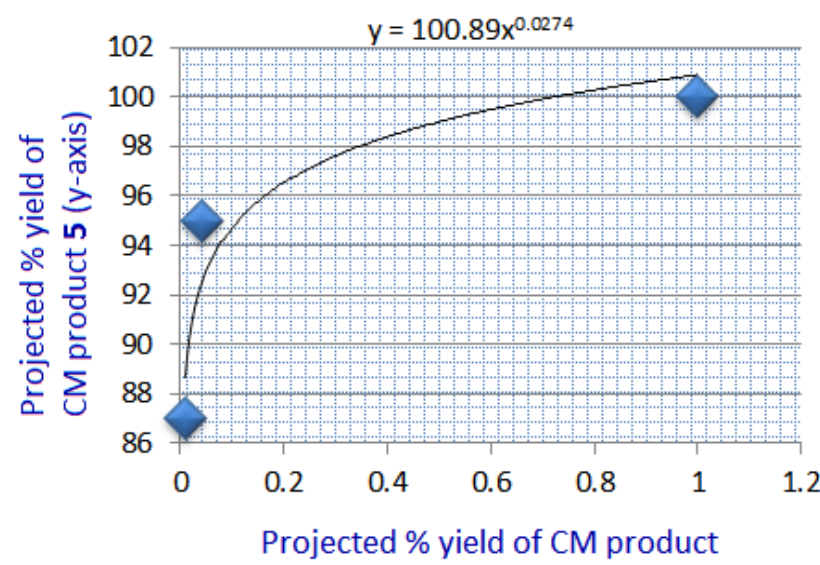

Figure 3. A graph of yield of styrene CM vs. mol.\% of catalyst 2 used.

Table 3. Theoretical extrapolation of the $\mathrm{CM}$ product at lower concentrations of catalyst 2 .

\begin{tabular}{|c|c|}
\hline Mol. \% of 2 & Yield $^{\text {c }}$ \\
\hline $1 \times 10^{-1}$ & 93.9 \\
\hline $1 \times 10^{-2}$ & 88.2 \\
\hline $1 \times 10^{-3}$ & 82.8 \\
\hline $1 \times 10^{-4}$ & 77.1 \\
\hline $1 \times 10^{-5}$ & 71.6 \\
\hline $1 \times 10^{-6}$ & 66.0 \\
\hline $1 \times 10^{-7}$ & 60.4 \\
\hline $1 \times 10^{-8}$ & 54.9 \\
\hline $1 \times 10^{-9}$ & 49.3 \\
\hline
\end{tabular}

${ }^{\mathrm{C}}$ Projected CM Yield calculated using $\mathrm{y}=100.89 \mathrm{x}^{0.0274}$

\subsection{Theoretical Extrapolation at Lower Catalyst Concentrations}

Though we are interested in determining the yield of CM at ultra-low concentrations of catalyst 2, we were barred by the challenges of associated with running reactions at ultra-low concentrations in our research laboratory. This requires weighing very low masses of catalyst $\mathbf{2}$ which increases the margin of error as the required weight approaches the error of the weighing instrument. To alleviate this problem, the other alternative is to use extremely large amounts of styrene which is beyond the scale/scope of our research laboratory. In order to circumnavigate this problem, we carried out theoretical determination based on the curve equation on Fig 3. The theoretical results generaed are reported in Table 4. Based on theoretical extrapolation, it can be seen that catalyst $\mathbf{2}$ is quite potent and and is expected to give reasonable yields at very low concentrations. The last entry on the table, shows that even at $1.0 \times 10^{-9} \mathrm{~mol} . \%$, the reaction is expected to give about $49 \%$ of the CM product. It is important to note that these results are only true when we assume that this equation is obeyed at ultra low concentrations. It is generally expected that at low concentrations, catalysts become inactive or are easily poisoned impurities, low-concentration inhibitors and competing reaction pathways.

\section{Conclusions}

This study has demonstrated that CM of styrene is feasible even at very low catalyst concentrations. The $\mathrm{CM} /$ homodimerization of styrene to afford trans-stilbene has been successfully carried out at reduced catalyst loading under aerobic conditions. The Grubbs $2^{\text {nd }}$ Generation catalyst 2 provided the best yields at lower concentrations in relatively short time. Catalyst $\mathbf{2}$ was found to be the most active under low concentrations followed by catalyst 4 . Further work needs to be done on $\mathrm{CM}$ of other alkene substrates and also on other metathesis transformations including ROM, ROMP and RCM reactions.

Most metathesis reactions are usually done under controlled inert environment. It will be interesting to see if the activity of the other catalysts 1,2 and 4 could be enhanced under inert gas atmosphere. Theoretical extrapolation projects that catalyst $\mathbf{2}$ will remain potent even at ultra-low concentrations. It will be intresting to see if these figures can be confirmed experiementally and also to determine the limits of the exponential expression, $\mathbf{y}=$ $100.89 x^{0.0274}$.

\section{Representative Procedure}

An oven dried round bottom flask was alowed to cool. The flask was then fitted with a magnetic stir bar, a rubber septum, a reflux condenser and a needle inserted through the rubber septum to maintain aeroboc conditions. Styrene ( $0.779 \mathrm{~g}, 7.40 \mathrm{mmol}$.) and dichloromethane $(75 . \mathrm{mL}$ ) were added to make a final concentration of $1.0 \mathrm{M}$ styrene (conc. of $0.5 \mathrm{M}$ if you consider that 0.5 eq. reacts with the other 0.5 eq. to give 1 eg. of stilbene). Grubbs $2^{\text {nd }}$ generation catalyst 2 (32 mg, $3.8 \times 10^{-5} \mathrm{mmol} ., 1 \mathrm{~mol}$. \%) was added in one portion and the resulting solution stirred at reflux for $3 \mathrm{~h}$. The color of the solution changed from brownish-red to pale yellow. The reaction mixture was taken out of the oil bath and the volume reduced in-vacuo then purified directly on a silica gel column $(2 \times 10 \mathrm{~cm})$, eluting with $100 \mathrm{~mL}$ of a 9:1 hexanes:ethyl acetate and the combined fractions 
concentrated to yield the stilbene product $(\mathrm{Rf}=0.7$ in 9.7:0.3 hexane : ethyl acetate) as colorless flakes $(0.670 \mathrm{~g}, 99 \%$ yield). ${ }^{1} \mathrm{H}$ NMR (400 MHz, $\left.\mathrm{CDCl}_{3}, \mathrm{ppm}\right): \delta 7.52(\mathrm{~m}, 4 \mathrm{H})$, $7.35(\mathrm{~m}, 4 \mathrm{H}), 7.25(\mathrm{~m}, 2 \mathrm{H}), 7.10(\mathrm{~s}, 2 \mathrm{H})\left(5 \mathrm{H} ;{ }^{13} \mathrm{C}\right.$ NMR $(100$ $\mathrm{MHz}_{\mathrm{CDCl}}$, ppm): $\delta 137.6,129.0,128.9,127.9,126.8$. The ${ }^{1} \mathrm{H}$ NMR and ${ }^{13} \mathrm{C}$ NMR Spectra matched with that reported in the literature. ${ }^{16,17}$

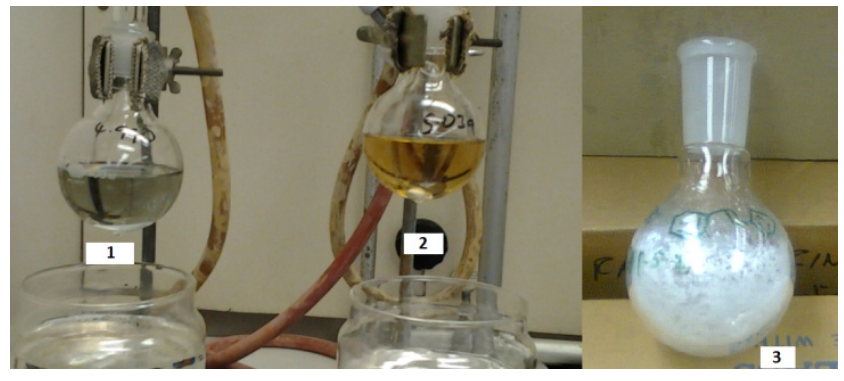

Figure 4. Appearance of the reaction mixtures after metathesis reaction using catalyst $\mathbf{3}$ (flask 1) and catalyst $\mathbf{1}$ (flask 2). Notice the faint yellow color of the dissolved trans-stilbene product. The third flask (flask 3 ) shows the product after column purification followed by solvent removal using rotary evaporator.

\section{Acknowledgements}

This manuscript is based on the work supported by National Science Foundation (NSF\# 1205608 and 1040094) and the Gallaudet Research Institute. Dr. Xueqing Song is acknowledged for helpful discussions and NMR data acquisition.

\section{REFERENCES}

[1] Schmalz, H.-G. Catalytic Ring-Closing Metathesis: A New, Powerful Technique for Carbon-Carbon Coupling in Organic Synthesis. Angew. Chem. Int. Ed. Engl. 1995, 34, 1833-1836 DOI: 10.1002/anie.199518331.

[2] Bajwa, N.; Jennings, M. P. Syntheses of epi-aigialomycin D and deoxy-aigialomycin $\mathrm{C}$ via a diastereoselective ring closing metathesis macrocyclization protocol. Tetrahedron Lett. 2008, 49, 390-393.

[3] Chatterjee, A. K.; Morgan, J. P.; Scholl, M.; Grubbs, R. H. Synthesis of functionalized olefins by cross and ring-closing metatheses. J. Am. Chem. Soc. 2000, 122, 3783-3784.

[4] Schrock, R. R. Olefin metathesis by molybdenum imido alkylidene catalysts. Tetrahedron 1999, 55, 8141-8153.

[5] Schwab, P.; France, M. B.; Ziller, J. W.; Grubbs, R. H. A Series of Well-Defined Metathesis Catalysts-Synthesis of $\left[\mathrm{RuCl} 2\left(=\mathrm{CHR}^{\prime}\right)(\mathrm{PR} 3) 2\right]$ and Its Reactions. Angew. Chem. Int. Ed. Engl. 1995, 34, 2039-2041 DOI: 10.1002/anie. 199520391.

[6] Sanford, M. S.; Love, J. A.; Grubbs, R. H. Mechanism and Activity of Ruthenium Olefin Metathesis Catalysts. J. Am. Chem. Soc. 2001, 123, 6543-6554 DOI: 10.1021/ja010624k.

[7] Toste, F. D.; Chatterjee, A. K.; Grubbs, R. H. Functional group diversity by ruthenium-catalyzed olefin cross-metathesis. Pure Appl. Chem. 2002, 74, 7-10.

[8] Woodward, C. P.; Spiccia, N. D.; Jackson, W. R.; Robinson, A. J. A simple amine protection strategy for olefin metathesis reactions. Chem. Commun. 2011, 47, 779-781.

[9] Hong, S. H.; Grubbs, R. H. Highly Active Water-Soluble Olefin Metathesis Catalyst. J. Am. Chem. Soc. 2006, 128, 3508-3509 DOI: 10.1021/ja058451c.

[10] Audic, N.; Clavier, H.; Maudit, M.; Guillemin, J.-C. An Ionic Liquid-Supported Ruthenium Carbene Complex: A Robust and Recyclable Catalyst for Ring-Closing Olefin Metathesis in Ionic Liquids. J. Am. Chem. Soc. 2003, 125, 9248-9249.

[11] Buijsman, R. C.; van Vuuren, E.; Sterrenburg, J. G. Ruthenium-Catalyzed Olefin Metathesis in Ionic Liquids. Org. Lett. 2001, 23, 3785-3787.

[12] Grela, K. Progress in metathesis chemistry. Beilstein J. Org. Chem. 2010, 6, 1089-1090 DOI: 10.3762/bjoc.6.124.

[13] Thayer, A. M. Making metathesis work. Chem. Eng. News 2007, 85, 37-47.

[14] Fihri, A.; Luart, D.; Len, C.; Solhy, A.; Chevrin, C.; Polshettiwar, V. Suzuki-Miyaura Cross-Coupling Coupling Reactions with Low Catalyst Loading: A Green and Sustainable Protocol in Pure Water. Dalton Trans. 2011, 13, 3116-3121.

[15] Dinger, M. B.; Mol, J. C. High turnover numbers with ruthenium-based metathesis catalysts. Adv. Synth. Catal. 2002, 344, 671 .

[16] Patel, J.; Mujcinovic, S.; Jackson, W. R.; Robinson, A. J.; Serelis, A. K.; Such, C. High conversion and productive catalyst turnovers in cross-metathesis reactions of natural oils with 2-butene. Green Chem. 2006, 8, 450-454.

[17] Anderson, D. R.; Ung, T.; Mkrtumyan, G.; Bertrand, G.; Grubbs, R. H.; Schrodi, Y. Kinetic Selectivity of Olefin Metathesis Catalysts Bearing Cyclic

(Alkyl)(Amino)Carbenes. Organometallics 2008, 27, 563566 DOI: $10.1021 /$ om7008028.

[18] Chatterjee, A. K.; Choi, T.-L.; Sanders, D. P.; Grubbs, R. H. A general model for selectivity in olefin cross metathesis. $J$. Am. Chem. Soc. 2003, 125, 11360-11370.

[19] Wheeler, O. H.; Pabon, H. N. B. de. Synthesis of Stilbenes. A Comparative Study1. J. Org. Chem. 1965, 30, 1473-1477 DOI: $10.1021 /$ jo01016a031.

[20] Amini, M.; Bagherzadeh, M.; Moradi-Shoeili, Z.; Boghaei, D. M. $\mathrm{Pd}(\mathrm{OAc}) 2$ without added ligand as an active catalyst for Mizoroki-Heck reaction in aqueous media. $R S C A d v$. 2012, 2, 12091-12095 DOI: 10.1039/C2RA21459H. 